\title{
Effective Proof Reading Strategies for Comprehending Mathematical Proofs
}

\author{
Keith Weber ${ }^{1}$
}

Published online: 3 July 2015

(C) Springer International Publishing Switzerland 2015

\begin{abstract}
In this paper, I identify five effective proof reading strategies that mathematics majors can use to comprehend proofs. This paper reports two studies. The first study is a qualitative study in which four successful mathematics majors were videotaped reading six proofs. These students used five proof reading strategies to foster comprehension: (i) trying to prove a theorem before reading its proof, (ii) identifying the proof framework being used in the proof, (iii) breaking the proof into parts or sub-proofs, (iv) illustrating difficult assertions in the proof with an example, and (v) comparing the method used in the proof with one's own approach. The qualitative analysis of these proof reading strategies illustrates how they may foster comprehension. The second study was a quantitative study in which 83 mathematics professors were surveyed about whether they desired that their students use these strategies. Most mathematicians indicated that they desired that students use them.
\end{abstract}

Keywords Proof Proof comprehension · Proof reading - Undergraduate mathematics education

In describing the nature of instruction in university mathematics courses, Davis and Hersh (1981) contended that "a typical lecture in advanced mathematics... consists entirely of definition, theorem, proof, definition, theorem, proof, in solemn and unrelieved concatenation" (p. 151). Similarly, Dreyfus (1991) wrote that the typical mathematics instructor teaches "almost exclusively the one very convenient and important aspect which has been described above, namely the polished formalism, which so often follows the sequence theorem-proof-application" (p. 27). While these descriptions probably exaggerate the homogeneity of mathematics instruction and the extent that proof dominates mathematics lectures, it is the case that much of the teaching in

Keith Weber

keith.weber@gse.rutgers.edu

1 Graduate School of Education, Rutgers University, 10 Seminary Place, New Brunswick, NJ 08901, USA 
advanced mathematics courses consists of the students observing the proofs that their professors present to them (e.g., Fukawa-Connelly 2012; Mills 2011; Weber 2004).

An important assumption behind this pedagogical practice is that students can learn mathematics by reading and studying these proofs. However, both mathematicians and mathematics educators question whether this assumption is true. The mathematician Carl Cowen (1991) addressed the issue as follows:

If you need evidence that we have a problem, let one of your B students ... explain the statement and proof of a theorem from a section in the book that you have skipped. My students, at least, do not have the innate ability to read and understand what they have read. When I ask them to read a problem and explain it to me, the majority just recite the same words back again (p. 50).

This passage is consistent with claims by mathematics educators that their students are frequently confused by the proofs that they read (e.g., Conradie \& Frith 2000; Porteous 1986; Rowland 2001). Empirical studies also illustrate mathematics majors' difficulties with reading proofs by demonstrating that students often cannot distinguish between valid and invalid arguments (Alcock \& Weber 2005; Ko \& Knuth 2012; Selden \& Selden 2003; Weber 2010). Consequently, mathematics majors are likely to learn little from the proofs that are presented to them.

While research studies have documented that mathematics majors do not read proofs effectively (e.g., Alcock \& Weber 2005; Inglis \& Alcock 2012; Ko \& Knuth 2012; Selden \& Selden 2003), there is little research on how students should read proofs to foster comprehension (for a notable exception, see Hodds et al. 2014, discussed later in this paper). The goal of this paper is to address this issue. Specifically I highlight five proof reading strategies, illustrate how they can help undergraduates comprehend the proofs that they read, and document that they are valued by most mathematics professors.

\section{Related Literature}

\section{Proofs as the Bearers of Mathematical Knowledge}

Building on the work of Rav (1999), Hanna and Barbeau (2010) argued that mathematicians do not only use proof to establish that a theorem is correct, but the proof itself contains mathematical knowledge beyond the theorem-statement that it establishes. In fact, most mathematicians claim their main reason for reading the published proofs of others is to look for insights within the proof that they can use to solve problems that they are considering (Mejía-Ramos \& Weber 2014; Weber \& Mejía-Ramos 2011). Hanna and Barbeau (2010) further argued that this is especially relevant in the mathematics classroom. Since students frequently accept mathematical assertions as true based on empirical evidence or an appeal to authority (e.g., their professor or textbook referred to the assertion as a theorem), proof should play a role in the classroom beyond providing a justification (see also de Villiers 1990; Hanna 1990).

Several interview studies have found that mathematics professors agree with Hanna and Barbeau (2010). In these studies, mathematicians report that their reasons for 
presenting proofs in the classroom are not so students will believe the theorems that are being proven, but to introduce new methods for proving and enhance understanding of the mathematical concepts being discussed (e.g., Nardi 2008; Weber 2012; Yopp 2011). However, these interviewed mathematicians found it difficult to balance the desire to enhance understanding with the disciplinary demands of presenting rigorous arguments and the practical constraints of working with struggling students (e.g., Lai \& Weber 2014; Lai et al. 2012; Nardi 2008). In particular, mathematicians claimed they were sometimes reluctant to use the informal language or representations that can enhance understanding since students were more prone to error when doing the same (Nardi 2011). Weber (2012) observed that even though the mathematicians in his interview study wanted their students to understand the proofs that were presented to them, these professors claimed that they usually did not discuss with students how they should be reading these proofs and they only assessed students' understanding of a proof in a superficial manner. Such findings suggest that outlining specific things that students can do to increase their understanding of the proofs that they read would be of practical use to mathematics professors.

\section{Literature on the Reading of Mathematical Proof}

In mathematics education, most empirical studies on the reading of proof can be broadly classified into two categories. In the first category, researchers ask students (or teachers) to evaluate different types of arguments against some criterion, such as whether they find the argument to be personally convincing or whether the argument would qualify as a proof. They then use the students' evaluations to infer what types of evidence they find convincing or what types of proof schemes (Harel \& Sowder 1998) that they hold. A key claim from this research is that some students are convinced a universal claim is true if it has been verified for several specific examples (e.g., Healy \& Hoyles 2000; Martin \& Harel 1989; Segal 2000). (However, my colleagues and I challenge whether it is appropriate to infer proof schemes based only on what students evaluate as "convincing". See Weber et al. 2014 and especially Weber and MejíaRamos 2015, for a detailed critique). In a second category of studies, researchers present students with ostensibly deductive proofs and ask them to determine whether or not these proofs are correct. The goal of these studies is for researchers to determine if students can distinguish between valid and invalid arguments and to understand the processes students use to make these judgments (e.g., Alcock \& Weber 2005; Inglis \& Alcock 2012; Ko \& Knuth 2012; Selden \& Selden 2003). This research revealed that mathematics majors have difficulty with proof validation tasks, in part because they focus largely on calculations within a proof while paying little attention to the proof's overarching structure (Inglis \& Alcock 2012; Selden \& Selden 2003).

In their literature reviews, both Mejía-Ramos and Inglis (2009) and Selden (2012) noted there have been few empirical studies on proof comprehension, urging for more work in this area. A notable exception is an important recent paper by Hodds et al. (2014). In this paper, the authors found that students who were trained to provide selfexplanations performed better on a proof comprehension test than those who were not. This effect was found in a laboratory setting, as well as in a classroom in which the treatment consisted of a brief fifteen minute "in lecture self-study training". Because their instructional treatment produces robust learning gains and their treatment is 
practical to implement and disseminate, Hodds, Alcock, and Inglis have made a valuable theoretical and practical contribution to the field. Nonetheless, in their studies, even after studying self-explanation, students' performance on proof comprehension tests was still far from ideal (scoring about $65 \%$ on the test in the laboratory setting, $60 \%$ in the classroom setting, in comparison to about $50 \%$ for the control group), suggesting that reading strategies specific to proof reading can still provide further benefits to students.

\section{Understanding a Proof in Advanced Mathematics}

To define what it means to understand a proof, I adopt the proof comprehension model of Mejía-Ramos et al. (2012). To form this model, Mejia-Ramos et al. interviewed nine mathematicians about what they hoped their students would gain from the proofs that they presented in their classrooms. Based on commonalities in mathematicians' responses, the authors identified seven facets of what it means for a mathematics major for to understand a proof. In their model, Mejía-Ramos et al. (2012) distinguished between a local understanding - where the understanding can be gleaned by studying a small number of statements (perhaps a single inference) within the proof- and a holistic understanding based upon the ideas or methods that motivate the proof in its entirety.

At a local level, understanding a proof would be comprised of (a) knowing the meaning of the terms and statements within the proof, including the meaning of the claim being proven, (b) being able to justify how new assertions in a proof followed from previous ones, and (c) identifying the proof framework (Selden \& Selden 1995) being used, such as a direct proof or proof by contradiction, and seeing how the assumptions and conclusions of the proof fit within this framework.

The research literature suggests that students often do not try to obtain this local understanding, at least when they are checking a proof for correctness. For instance, Selden (2012) reported that students often did not understand the theorem statement before they read the proof (see also Conradie \& Frith 2000). Inglis and Alcock (2012) reported that, in contrast to mathematicians, mathematics majors often did not appear to justify how new assertions in a proof followed logically from previous ones. Selden and Selden (2003), Inglis and Alcock (2012), and Weber (2010) found that most students would accept a proof of a conditional statement even if it began by assuming the conclusion and deducing the antecedent, which suggests that these students were not carefully considering the validity of the proof technique being applied. However, it should be noted that each of the studies cited above asked students to validate proofs rather than understand them.

In the Mejía-Ramos et al. (2012) proof comprehension model, the holistic understanding of a proof consists of being able to: (a) provide a summary of the proof that emphasizes its high level goals, (b) apply the methods of the proof in other situations to prove new theorems, (c) break the proof into its main parts or sub-proofs, and (d) apply the methods of the general proof to a specific example (e.g., if one read a proof that a perfect square has an odd number of factors, use the ideas of the proof to explain why 36 must have an odd number of factors).

There have been few systematic studies on mathematics majors' holistic understanding of the proofs that they read, although Conradie and Frith (2000) and Cowen (1991) have remarked that in their experience, students have performed poorly when asked 
questions of this type. Selden and Selden (2003) and Inglis and Alcock (2012) found that when mathematics majors validate proofs, they focus on line-by-line checks rather than studying the overarching methods used in the proof. Weber and Mejía-Ramos (2014) reported that most mathematics majors do not conceptualize a proof holistically; instead they viewed understanding a proof as tantamount to understanding how each new assertion followed from previous ones, which suggests that mathematics majors predominantly focus on developing a local understanding.

\section{Transparency of these Facets of Understanding in Proofs}

Building on Hemmi's (2008) notion of the transparency of a proof, Hemmi and Jaworski (2009) treated a proof as a cultural artifact. They distinguished between aspects of a proof that are visible to students, meaning these aspects of the proof received explicit emphasis, and that were invisible to students, where a particular aspect of the proof is ignored and would not be transparent to a student. In traditional proofs, most of the facets of understanding in Mejia-Ramos et al.'s (2012) model are invisible to the students. For instance, Leron (1983) and Anderson, Boyle, and Yost (1986) observed that the linear deductive presentation of proofs masks the high-level ideas contained in the proof, making a proof difficult to summarize, with Leron (1983) further noting that some of the ideas in the proof may appear unmotivated, making it challenging for a student to apply the ideas in the proof in another setting. Also, the justification for how new assertions in a proof follow from previous ones are often left implicit, leaving the reader to infer what general mathematical principles are being used to make these deductions (Alcock \& Weber 2005; Weber \& Alcock 2005).

One suggestion to address this pedagogical problem is to use alternative formats for suggesting proofs that make aspects of proof understanding visible to students (e.g., Alcock 2009; Leron 1983; Leron \& Zaslavsky 2009; Rowland 2001). For instance, Leron (1983) proposed using structured proofs that makes the summary of the proof and the motivation for using certain techniques visible to the students. Unfortunately, recent reviews of the literature have either reported that there are few empirical studies demonstrating the effectiveness of these alternative proof formats (e.g., Dreyfus et al. 2012; Selden 2012) or concluded that the existing empirical evidence suggests that these formats are ineffective for improving comprehension (Fuller et al. 2014; Hodds et al. 2014). Hodds et al. (2014) concluded that the key to having students likely does not involve making important ideas of the proof visible to the students, but rather to have students productively engage in the proof so students could perceive the important aspects of the proof that are invisible to them. The goal of this paper is to discuss reading strategies that students can use to accomplish this.

\section{Study 1: Analyzing Successful Students' Proof Reading Strategies}

\section{Research Methods}

Goals of this Study Because research in proof comprehension is sparse in the mathematics education literature (Mejía-Ramos \& Inglis 2009), I chose to conduct an initial exploratory study to generate potentially effective proof reading strategies that may 
foster proof comprehension. To identify such strategies, I strove to record an environment where they were likely to be elicited. For this reason, I chose to video-record articulate successful mathematics majors as they thought aloud while reading six proofs from calculus and basic number theory. My rationale was that successful students were likely to exhibit productive strategies and articulate students were likely to vocalize their thinking clearly, enabling the researcher to recognize these strategies when they were used.

To avoid misinterpretation, I will delineate what I expect to generalize from this qualitative study (Firestone 1993). I am not attempting sample-to-population generalization; that is, I am not claiming or hypothesizing that all or most successful mathematics majors use the strategies that I report from Study 1. Rather, the purpose of Study 1 is to develop grounded hypotheses about what strategies can be useful to foster proof comprehension. The important evidence in the development of these hypotheses is not the fact that some successful students used them but rather the theoretical rationales for their effectiveness that I provide and illustrate with episodes of these students' behavior.

Participants This study took place at a large state university in the northeast United States. Four students participated in this study. The students were each given a modest payment in exchange for their participation. All four participants were mathematics majors in their senior year at the time of the study; they were also concurrently enrolled in the mathematics teacher education program at that university and preparing to become high school mathematics teachers.

These four participants were chosen for several reasons. In their mathematics education courses, these students displayed an interest in mathematics and an eagerness to learn it, which suggested that they would take the proof reading tasks seriously. They were highly successful in their content-based mathematics education courses, ${ }^{1}$ suggesting that they would have the mathematical expertise to employ effective proof reading strategies. All four students had previously participated in other mathematics education research studies at the university where this study took place (none of these studies concerned proof reading) and in these studies, these students were both articulate and willing to share their reasoning. This increased the likelihood that these students would provide useful data. I make no claims that these participants are representative of all mathematics students. On the contrary, these students were chosen because they were not representative in that they were expected to employ effective proof reading strategies that most mathematics majors did not use.

Materials All materials can be found in the Appendix of this paper. The materials consisted of six theorem-proof pairs. Throughout the remainder of the paper, I refer to the $N^{\text {th }}$ theorem that the participants saw as Theorem $N$ and its associated proof as Proof $N$. These proofs were designed to have the following features: (a) the mathematical content of the proof relied on introductory calculus and basic number theory (so a lack of content knowledge presumably would not be a barrier to proof comprehension), (b)

\footnotetext{
${ }^{1}$ I am not aware of the grades they earned in their mathematics courses. I initially conjectured that these students were "successful" because they exhibited desirable attributes in their mathematics education courses and earlier studies. This conjecture that they were successful at reading proofs for comprehension was confirmed when they could answer the questions about the proofs from this study.
} 
the proofs were of interesting propositions whose veracity would not be immediately obvious to an undergraduate student, and (c) the proof employed an interesting proof technique with which I expected the participants would not have had extensive experience. For each proof, I generated a set of proof comprehension questions using the proof comprehension model put forth by Mejía-Ramos et al. (2012).

Procedure Participants met in pairs with the author of this paper for a task-based interview. The decision to interview the students in pairs was made for two reasons. First, I had conducted an earlier study (Weber 2010) in which participants were asked to "think aloud" while individually validating proofs and found the verbal protocols from the students were sparse and tended not to yield useful data. Second, Schoenfeld (1985) and Alcock and Simpson (2004) claimed that having students work on mathematical tasks in pairs leads to conversation between students that produces data that is both richer and more authentic than students working in a laboratory task in isolation. I assigned the first pair of students that were interviewed the pseudonyms Kevin and Tim and the second pair of students the pseudonyms Caleb and Derek.

Each pair of participants met with me for a video-recorded task-based interview. In the interviews, the pair of students were presented with a proof and asked to "think aloud" as they read and studied the proof. They were asked to read the proof until they felt that they understood it and they were told that they would be asked a series of questions about the proof after they had read it. When the pair felt they understood the proof, the interviewer took the proof from the students and presented them with a series of comprehension questions, one at a time, that were generated from the proof assessment model of Mejía-Ramos et al. (2012). In this study, both pairs of participants answered nearly every question correctly, with each pair providing satisfactory answers to all but two questions across the six proofs. This high rate of success was unusual (for comparison, see Fuller et al. 2014) and suggested that the participants understood the proofs that they had read. After answering these questions, if the interviewer had observed the participants using what he perceived to be an interesting proof reading strategy, he would ask the participants if they regularly used this strategy when reading proofs and what benefits they felt it provided for them. This process was then repeated for each of the six proofs.

Analysis As there are few empirical studies on proof comprehension (Mejía-Ramos \& Inglis 2009), I did not have any pre-existing categories in mind when analyzing this data and opted to use an open coding scheme in the style of Strauss and Corbin (1990).

In a first pass through the data, a graduate assistant and I noted each attempt that the pair of participants used to make sense of the theorem or the proof, where an "attempt" involved anything beyond a literal reading of the text and the graduate student and I then constructed a summary of the participants' behavior. After this, I sorted similar "attempts" into categories of strategies and gave the strategy a name and a definition. I then went through the data explicitly coding for every instance of the use of that strategy.

For each strategy that I generated, I studied instances of its implementation, as well as participants' comments on why they believed that strategy was beneficial to them, to see if I could hypothesize a theoretical rationale for why the strategy would be useful for proof comprehension. I mapped how each strategy helped the participants gain the 
components of understanding specified in the Mejía-Ramos et al. (2012) proof comprehension model. Using this process, I identified six potentially effective proof reading strategies.

\section{Results}

Tables 1 present a summary of the strategy usage for both pairs of participants.

\section{Strategy \#1: Understand the Theorem Statement}

Both pairs of students would invest time in trying to understand the theorem statement prior to reading its proof. The participants in this study sought to gain this understanding in two ways. First, participants would sometimes rephrase the theorem they were studying in their own words. This was illustrated when Kevin and Tim read Theorem 3, which introduced the concept of $k$-tuply perfect numbers. The theorem statement was:

We say that $n$ is $k$-tuply perfect if and only if $\sigma(n)=\sum_{d / n} d=k n$.

Theorem 3 If $n$ is 3-tuply perfect and 3 does not divide n, then $3 n$ is 4-tuply perfect.

After spending some time trying to figure out exactly what was being asserted, Kevin became frustrated and suggested reading the proof.

[1] Kevin: Well we just have to agree with the proof.

[2] Tim: Yeah, but we have to figure out what it [Theorem 3] is saying, so hold on.

Table 1 Student pairs' strategy usage for each proof

\begin{tabular}{|c|c|c|}
\hline Strategy & $\begin{array}{l}\text { Proofs where this } \\
\text { strategy was used } \\
\text { by Kevin and Tom }\end{array}$ & $\begin{array}{l}\text { Proofs where this } \\
\text { strategy was used } \\
\text { by Caleb and Derek }\end{array}$ \\
\hline $\begin{array}{l}\text { 1a. Understanding the theorem by rephrasing } \\
\text { it in one's own words }\end{array}$ & $2,3,5$ & 3,6 \\
\hline $\begin{array}{l}\text { 1b. Understanding the theorem by expressing } \\
\text { it in logical notation }\end{array}$ & 4 & 4 \\
\hline $\begin{array}{l}\text { 2. Trying to prove a theorem before reading } \\
\text { its proof }\end{array}$ & $1,2,3,4,5,6$ & $2,5,6$ \\
\hline $\begin{array}{l}\text { 3. Considering the proof framework used in } \\
\text { the proof }\end{array}$ & 2,5 & $2,3,5$ \\
\hline 4. Partitioning the proof into parts or sub-proofs & $3,5,6$ & $1,2,5$ \\
\hline 5. Checking confusing inferences with examples & 3,5 & 3,4 \\
\hline $\begin{array}{l}\text { 6. Comparing the method used in the proof } \\
\text { with one's own approach }\end{array}$ & & 1 \\
\hline
\end{tabular}


In the passage above, Tim insisted that the pair understand Theorem 3 prior to reading Proof 3. To build this understanding, Kevin reformulated Theorem 3 less formally using his own words.

[3] Kevin: So if $n$ is 3-tuply perfect, so that's if all the divisors including itself add up to $3 n$, and if that's the case, and 3, just the number 3, does not divide whatever $n$ is, then $3 n$, then 3 times it is 4 -tuply perfect.

There are two noteworthy phenomena in this interaction. First, observe that in [1], Kevin was about to abandon his quest to understand the theorem statement, even though he subsequently demonstrated that he was capable of doing this in [3]. It was only Tim's directive in [2] that compelled Kevin to develop this understanding. Second, not only did Kevin's interpretation of the theorem statement in [3] describe what the theorem meant, it also gave an indication of how the proof might be constructed by stating what might be assumed and what should be proven in a direct proof.

In other instances, both pairs of students would try to express the theorem in terms of first order logic. For instance, when Caleb and Derek read Theorem 4, which asserts that there is a real number whose fourth power is exactly one more than itself, they jointly constructed a precise logical formulation of what this meant:

[1] Derek: So the theorem is, "there is a real number whose fourth power is exactly one larger than itself".

[2] Caleb: [writing] So there exists an $\mathrm{x}$ in the reals such that $x$ to the fourth is...

[3] Derek: equal to $x+1$.

[4] Caleb: exactly one...

[5] Derek: One larger than itself. Right?

[6] Caleb: Alright.

[7] Derek: Alright.

[Caleb had written $\left.\exists x \in R\left(x^{4}=x+1\right)\right]$.

In this episode, Derek and Caleb have co-constructed what it would mean to understand Theorem 4 symbolically, which permits algebraic ways of thinking about the theorem.

\section{Strategy \#2: Try to Prove the Theorem Statement Before Reading its Proof}

Before reading each of the six proofs, Tim and Kevin would first try to prove the theorem themselves before reading its proof. It was only when it was not obvious to them how to make further progress that they read the proof of the theorem. The benefits of this are illustrated in the following excerpt, where Tim and Kevin read Theorem 1, which asserts that " $4 x^{3}-x^{4}+2 \sin x=30$ has no solutions".

[1] Tim: As $x$ gets really big, it gets dominated by the negative $x$ to the fourth term. And it's a parabola going down basically and it's going to get modulated a little bit.

[2] Kevin: Right. And sine of $x$ is...

[3] Tim: Periodic. 
[4] Kevin: It is periodic so that wouldn't really affect it too much... out of the two functions, $f(x)$ is the trumping one. [Proof 1 defines $f(x)=4 x^{3}-x^{4}$ ].

[5] Tim: So in the long run, it's going...

[6] Kevin: It's really $f(x)$ that matters.

[7] Tim: And the question is, does it reach 30.

In the excerpt, Kevin and Tim were unable to arrive at a proof. However, Kevin and Tim developed an idea for how a proof might proceed. In [3] and [4], Kevin and Tim observed that since $2 \sin x$ is periodic (or, more accurately, as an unstated consequence of $2 \sin x$ being continuous and periodic, $2 \sin x$ is bounded). In [5] through [7], they used this discovery to deduce that the key to the proof is showing that $4 x^{3}-x^{4}$ has a sufficiently low upper bound. The logical and algebraic details in the actual proof (Proof 1), such as using differentiation to find the critical points of $f(x)=4 x^{3}-x^{4}$, can be seen as supporting the high-level goals of finding bounds on each of the two functions. Consequently, when reading the proof, Kevin and Tim did not view the proof as an arbitrary series of inferences and calculations. Rather, their previous attempt at trying to prove the theorem made transparent to them that Proof 1 could be viewed as satisfying two high level goals - finding the bounds of $2 \sin x$ and $4 x^{3}-x^{4}$. Thus, their work allowed Kevin and Tim to recognize that the actual proof had two independent sub-parts and to provide a summary of the proof.

After reading all six proofs, Kevin and Tim were asked if they often tried to prove theorems before reading their proofs and, if so, why they did so. Kevin and Tim both agreed this was something that they did regularly and Tim listed several benefits for doing this. These benefits included "understanding the tricks and techniques so I can use them again" (corresponding to using the proof to identify methods that might be useful for proving other theorems), making him a more active proof reader, and providing extra practice with writing proofs.

\section{Strategy \#3: Considering the Proof Framework Used in the Proof}

When reading the proofs, Kevin and Tim sometimes explicitly attended to what claim (or sub-claim) was being proven, what proof technique was being employed, and what can be assumed and what must be shown using that proof technique. Kevin and Tim's attention to proof frameworks was most clearly illustrated in their reading of Proof 5, where Tim was confused by the overarching structure of the proof. Kevin explicitly attended to each of the issues described above, as is illustrated both in their conversation and their written work on Proof 6 presented in Fig. 1.

[1] Kevin: From [lines] two to four, it's doing the proof by contradiction. Suppose $n$ is not a perfect square. [...]

[2] Tim: Suppose $n$ is not a perfect square. So you're saying, suppose not this?

[3] Kevin: For which one?

[4] Tim: So you're saying, suppose $n$ is not perfect, right, and that's the opposite of the right side.

[5] Kevin: Cause it's dichotomous. If it's not a perfect square, then it's even. So therefore if $\mathrm{P}$ implies Q, then not Q implies not P, right? Contrapositive. 
[6] Tim: So we're saying this is $\mathrm{P}$ [writes $\mathrm{P}$ above "the number of divisors of a positive integer $n$ is odd"], this is Q [writes Q over " $n$ is a perfect square"].

[7] Kevin: OK sure. So $\mathrm{P}$ is a positive integer, right? Positive integer?... Right?... Positive integer?

[8] Tim: Is odd?

[9] Kevin: Right. Sorry. P is odd, right? So then not P would be even. So Q would be a perfect square, $\mathrm{k}$ squared. And not $\mathrm{Q}$ would not be $k^{2}$ or whatever. So here, [referring to lines 3-5 of Proof 5] it shows not Q implies not P.

[10] Tim: And this part of the proof ends at 5 [Tim draws horizontal dashed between lines 2 and 3 and between lines 5 and 6 to partition the two parts of the proof]

[11] Kevin: So it shows not Q implies not P and therefore P implies Q.

[12] Tim: OK.

[13] Kevin: So it's proving it forwards. [Tim rights a right arrow next to lines 3, 4, and 5]. It's the forwards way. [Reading line 6] On the other hand, suppose $\mathrm{n}$ is a perfect square. So now it's going to prove the backwards.

The fact that Proof 6 contained two sub-proofs, one of the sub-proofs was a direct proof (lines 6-8) and the other was a proof by contradiction (lines 3-5), was not visible in the proof. Indeed, only the subtle clause "on the other hand" to begin line 6 signaled that a new sub-proof was being written. We see Kevin and Tim providing a rather literal interpretation of how attending to the proof framework of the proof could make these features of Proof 6 visible. From lines [1] through [4], Kevin and Tim appear to have difficulty understanding each other. One issue concerns the confusion of what is being negated, which Tim in [4] called the right side. To resolve these difficulties in communication, Kevin in [5] established what $\mathrm{P}$ and $\mathrm{Q}$ are for the remainder of their interaction and the two proceeded to negotiate what not $\mathrm{P}$ and what not $\mathrm{Q}$ would represent. This focus on the meaning of $\mathrm{P}, \mathrm{Q}$, not $\mathrm{P}$, and not $\mathrm{Q}$ allowed the two to perceive where each sub-proof begins and ends, what each sub-proof is establishing, and what proof method (proof by contradiction, direct proof) is being used for each sub-proof.

\section{Strategy \#4: Partitioning the Proof into Parts or sub-Proofs}

For proofs that could be broken into independent sub-proofs, both pairs of students would sometimes partition the proof to see where one part of the proof began and the previous part ended. This was illustrated when discussing Strategy 3 above and in Fig. 1, where Kevin and Tim partitioned Proof 5 into the part that showed the "if" direction and the part that showed the "only if" direction to establish the logical equivalence claimed in Theorem 5. To further illustrate this strategy, consider how Kevin and Tim read Proof 3. The first eight lines of this proof consisted of proving the lemma "if $m$ and $n$ are relatively prime, then $\sigma(m) \sigma(n)=\sigma(m n)$ " and the last three lines of the proof were applying that lemma to conclude Theorem 3 . When reading the proof, Tim suggested partitioning the proof into parts and initially skipping the proof of the lemma.

[1] Kevin: Is this [the proof] of the lemma?

[2] Tim: This is the proof of the lemma.

[3] Kevin: Where does the proof of the lemma end? 


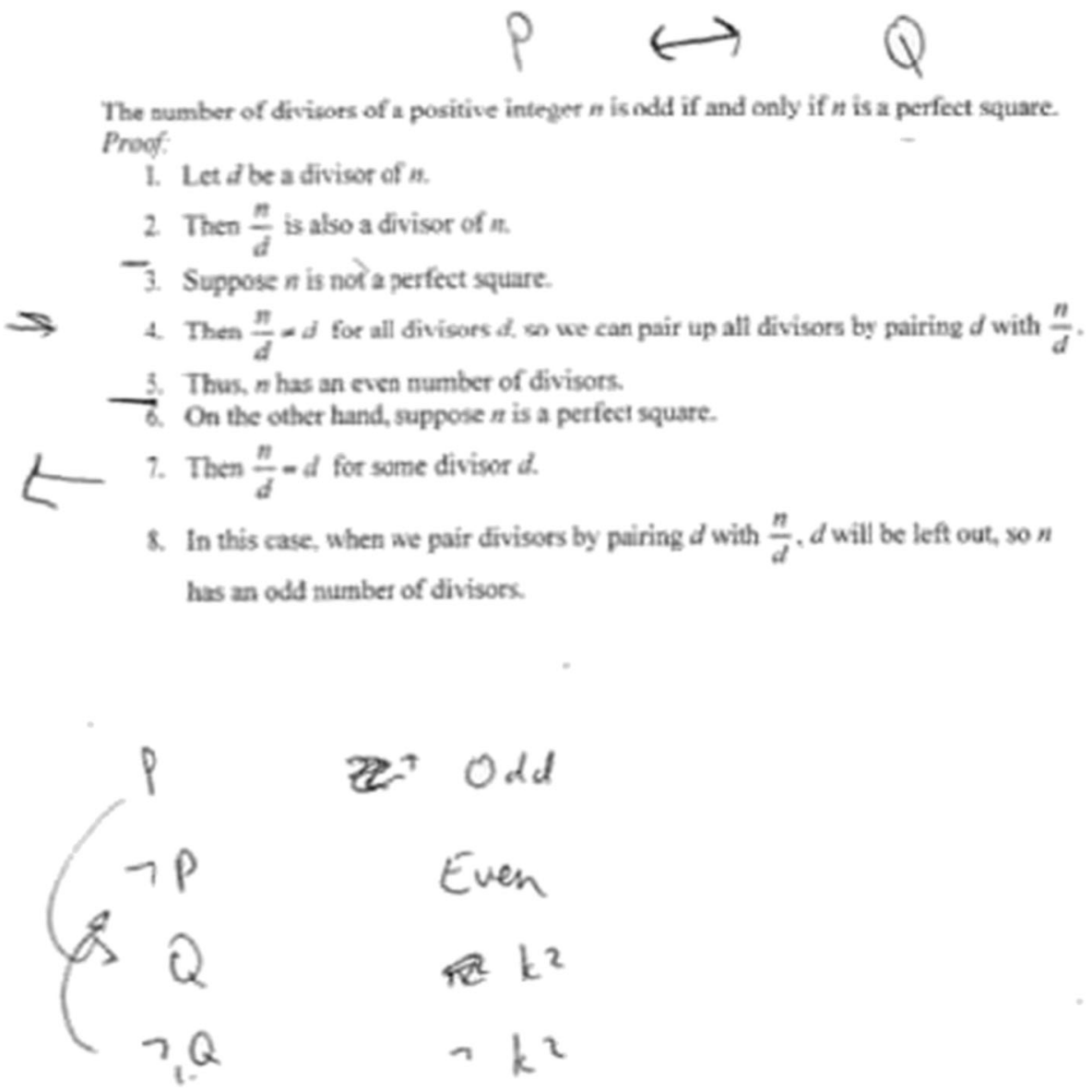

Fig. 1 Kevin and Tim's written work on Proof 6

[4] Tim: Good question.

[5] Kevin: Is there more? [checks the other side of the sheet]

[6] Interviewer: The proof of the lemma ends at step 8.

[7] Kevin: So why isn't this just the entire proof?

[8] Tim: Because when you use the lemma, you prove this.

[9] Kevin: So the actual proof is just like two steps. Well three steps. Well, nine to eleven, so it's four steps. OK. [Kevin begins reading the proof, starting at the lemma]

[10] Tim: So, let's not read the lemma first.

[11] Kevin: You have to read the lemma.

[12] Tim: Let's read the lemma later. Trust me.

The high-level structure of Proof 3 was not visible. The clearest indication that the lemma in line 1 was established was given at the start of line 9, which began, "with this lemma in hand". There was no clear statement in line 8 that indicated the proof of the lemma was completed. Upon reading the proof, Kevin and Tim immediately sought to find where the proof of the lemma was concluded (see [3]). In [5], Kevin was initially 
surprised that using the lemma made the proof so short (seeing if the proof proceeded on the other side of the paper), but in [8], Tim recognized that the proof was essentially an application of the lemma. Tim's insistence in line [12] that they read the lemma later allowed them to focus on the high level structure of the proof (what the lemma was and how it established the claim) and avoid becoming initially mired in the technical details of the proof (how the lemma was established). Later, Kevin claimed a benefit of partitioning the proof into parts was that it helped break complicated proofs into chunks that were manageable for him.

\section{Strategy \#5: Using Examples to Make Sense of Statements Within the Proof}

When there was confusion about an inference within a proof, both groups of participants would sometimes attempt to resolve this confusion by seeing how the step applied to a specific example. I illustrate this in the context of Caleb and Derek wrestling with the meaning of line 4 in Proof 3 . Lines 3 and 4 of Proof 3 are:

3. Then the divisors of $n$ are all numbers of the form

$$
d=p_{1}^{b 1} p_{2}^{b 2} \cdots p_{k}^{b k}, \text { where } 0 \leq b_{i} \leq a_{i}
$$

4. But these numbers are precisely the terms in the expansion of the product

$$
\left(1+p_{1}+p_{1}^{2}+\ldots+p_{1}^{a_{1}}\right)\left(1+p_{2}+p_{2}^{2}+\ldots+p_{2}^{a_{2}}\right) \ldots\left(1+p_{k}+p_{k}^{2}+\ldots+p_{k}^{a_{k}}\right)
$$

Upon reading lines 3 and 4, both students expressed confusion for why Line 4 should follow from Line 3 . To try to understand the situation better, they considered the specific case where $n=24$.

[1] Caleb: Say it's 24. 24 is 12 times 2, 6 times 2 times 2 so...

[2] Derek: 2 to the third times 3.

[3] Caleb: 2 to the third times 3. OK. 2 to the third times 3 is [pauses and refers to the formula in line 4 of Proof 3] our prime factorization. Right?

[4] Derek: Right.

[5] Caleb: So 3 is a sub 1. So they're saying that this is $1 \ldots$ [pause]

[6] Derek: Plus

[7] Caleb: Plus $p$ sub 1, so 2

[8] Derek: 2 plus 2 squared plus 2 cubed

[9] Caleb: Plus 2 squared plus 2 to the third times... [pauses and refers to the formula in line 4 of Proof 3]... 1 plus 3.

[10] Derek: I follow you there. So that's what that says.

[11] Caleb: [Expanding the product of $\left(1+2^{1}+2^{2}+2^{3}\right)(1+3)$ ] So that's 1 plus 3 plus 2 times 1 plus 2 times 3 plus 2 squared times 1 plus 2 squared times 3 plus...

[12] Derek: Why don't you just add these up? [Derek is referring to the terms of the expansion of $\left.\left(1+2^{1}+2^{2}+2^{3}\right)(1+3)\right]$.

[13] Caleb: Because I feel like I want to keep them...

[14] Derek: Oh, I see. Alright. I thought you were just trying to find the numbers so you can use them for the example. 
[15] Caleb: [Reading line 4 of Proof 3] But these numbers are precisely the terms of the expansion [Derek begins computing the sum of the divisors] It doesn't matter what the sum is though. Because what they are saying is that each of these terms are precisely the numbers they were talking about up here [pointing to line 3 of Proof 3] when they said these are all of the divisors. This is every single [pause]

[16] Derek: Factor?

[17] Caleb: Factor of 24.

[18] Derek: Because there's 1, there's 2, there's 3, there's 6, there's 4, there's 12, there's 24 , and there's 8 . I hit them all. 24 should have 8 factors. 1, 2, 3, 4, 5, 6, 7, 8. Hey, that's kind of cool! This is literally just adding up all the factors. Just like what we did up here [referring to line 3 of Proof 3]. So this is just a fancy way of saying this. This mumbo-jumbo down here is a fancy way of adding up all the factors. Okay, I see where this is going. This is starting to make sense to me.

In the excerpt above, Caleb and Derek instantiated Lines 3 and 4 of the proof with the example of $n=24$. It is interesting to note that from [1] through [15], Derek and Caleb appeared to be using $n=24$ for different purposes. Derek seemed to be using $n=24$ to empirically verify that the statement held, as can be seen in [12] where Derek suggests simplifying Caleb's expression by adding the terms and again in [15] when Derek begins adding the factors that Caleb identified. Caleb was using $n=24$ as a generic example, not allowing Derek to prematurely add the factors in line [13] and emphasizing "it doesn't matter what the sum is though" in line [15]. That the sum does not matter is a clear indicator that Caleb is not merely performing a verification (indeed, it doesn't matter that the result is actually verified) but he wants to see why this is true in the case of $n=24$. In [18], Derek revealed that Caleb's argument made transparent a fact that was invisible in the proof- the reason why line 4 followed from line 3.

\section{Strategy \#6: Comparing the Method in the Proof to one's own Methods}

After reading Proof 1, Caleb and Derek both remarked that the approach taken in the proof is not one that they would have chosen. The conversation given in the transcript below occurred immediately after reading Proof 1 .

[1] Caleb: Definitely not the way I would have gone when I looked at it...

[2] Derek: Not even close.

[3] Caleb: Yeah, I would not have broken it down like that.

[4] Derek: I would not have broken it down like that at all. How I would have solved this, I have no idea.

[5] Caleb: Well when you start putting in trigonometric functions.

[6] Derek: Yeah, that's when it starts getting a little hairy.

After this occurred, Caleb and Derek studied Proof 1 further and concluded their discussion with the following comments:

[7] Derek: I probably would never have ever thought of this but from my background in calculus, it makes sense to me.

[8] Caleb: Yeah, yeah. It's clever [laughs] 
[9] Derek: Yeah, it's clever. I never would have dreamed of coming up with something like this.

In [3] and [4], we see Caleb and Derek paid particular attention to the method used in Proof 1 (namely breaking the proof into two parts, each of which can be bounded) and in [5] and [6], they discussed why this technique was necessary (i.e., equations with polynomials and trigonometric functions cannot be solved algebraically). Hence, their discussion made the method used in the proof, including its novelty and its utility, an explicit object of discussion. At the end of the interview, the interviewer asked them if comparing the method in the proof to what they would have done was a proof reading strategy they sometimes employed and why they did so. In the excerpt below, Derek revealed that he uses this strategy since it helps him identify methods that might help him prove other theorems.

[10] Interviewer: This was something interesting that I've observed with the first proof that you looked at. So one of the things that you guys were marveling at was saying, "Oh, this technique is clever. This isn't the way that I would have approached it". Is that something that you do sometimes when you read a proof when you see a new technique?

[11] Derek: Yeah [while Caleb nods his head affirmatively]

[12] Int: Is there any benefits [sic] to doing that?

[13] Derek: Yeah, it gives you new techniques to solve proofs like that.

[14] Caleb: [nodding his head] Yeah.

\section{Summary}

In this study, I identified six strategies that the two pairs of successful students used to comprehend the six proofs that they read. Understanding the theorem statement not only enabled the participants to understand the meaning of one of the claims in the proof, but it also helped them understand the proof framework that would be used to establish the theorem. Trying to prove the theorem before reading its proof provided motivation for reading the proof as well as anticipating the proof framework that would be employed and how the proof was structured. Considering the proof framework in the proof clearly helped students understand why the assumptions and conclusions of the proof were appropriate. Breaking the proof into parts or sub-proofs not only enabled participants to see how the proof could be partitioned but also to see how the proof could be summarized. Instantiating specific claims in the proof helped students see how the proof applied to a specific example in addition to being able to understand statements within the proof and to see how they were justified. Comparing the method in the proof to one's own approach helped with identifying methods that might be useful in proving other theorems.

Strategy \#3 (considering proof frameworks) and Strategy \#5 (checking confusing statements in a proof with specific examples) have been suggested in the mathematics education literature (for instance, see Selden \& Selden 1995 and Weber 2008). However, the other strategies- notably Strategy \#2 (trying to prove a theorem before 
reading its proof) and Strategy \#6 (comparing the approach in the proof to one's own approach)- have received little to no emphasis in the literature to date.

\section{Study 2: A Survey on Mathematicians' Perceptions of the Desirability of These Strategies}

\section{Research Methods}

Goals of this Study In Study 1, I identified six strategies that successful students used to comprehend the proofs that they were reading and I provided a theoretical account for why these strategies are useful. The goal of the Study 2 is to document that mathematicians desire that students use these strategies.

An Internet-Based Study Following the methodology employed by Inglis and MejiaRamos (2009), I collected data through the internet in order to maximize the sample size of mathematicians. Recent studies have examined the validity of internet-based experiments by comparing a series of internet-based studies with their laboratory equivalents (e.g., Kranz \& Dalal 2000; Gosling et al. 2004). The notable degree of congruence between the two methodologies suggests that, by following simple guidelines, including checking the IP addresses and timestamps to ensure there were not multiple submissions from the same computer and checking that there are not significant differences on the responses of participants who complete the study and participants who do not, internet data has comparable validity to more traditional data. Given these safeguards and the impracticality of obtaining a large sample of mathematicians in any other fashion, I believe the use of an internet study is justified.

Participants To recruit participants, an e-mail was sent to the mathematics department faculty secretary at a large state university in each of the 50 states in the United States, asking them to forward an e-mail to the mathematics department, inviting mathematicians who had taught a proof-oriented course to participate in a survey on how they would like their students to behave in these courses. They were given a hyperlink to click on if they were interested in participating. Upon clicking on that link, participants were asked for demographic information, including the questions, "Have you ever taught a mathematics course where your students were asked to read and write proofs regularly?" If a participant answered no to this question, his or her responses were not included in the data to be analyzed. There were 83 participants $^{2}$ who indicated that they had taught such a course and completed every survey item.

Materials and Procedure For each strategy generated from Study 1, I generated a survey question (For one strategy, understanding the theorem before reading the proof, two survey questions were generated to account for the different ways this strategy was implemented in Study 1). For each strategy, mathematicians were shown two

\footnotetext{
${ }^{2}$ I am not aware of how many secretaries actually forwarded this message to their faculty, so I cannot estimate how many eligible mathematicians received this e-mail or what percentage of eligible mathematicians chose to participate.
} 
statements and asked to choose which statement they agreed with. One statement indicated that they preferred that mathematics majors use the strategy in question; the other statement indicated that they preferred that mathematics majors not use this strategy and then either provided a rationale for why they might not use this strategy or proposed an alternative strategy. This was done to make the foils sound plausible to the participants. (While it is debatable if the foils were sufficiently strong to sound plausible to mathematicians, in a separate study, my colleague and I found that most surveyed mathematics majors selected the foils for most survey items (Weber \& MejíaRamos 2013)). For instance, for the strategy of trying to prove a theorem before reading its proof, I generated the following two choices for participants:

A. When reading a proof of a theorem, I would prefer if mathematics majors think about how they might prove the theorem themselves before reading the proof.

B. I would prefer that mathematics majors not try to prove a theorem themselves before reading its proof. A reason for reading the proof is to see why the theorem is true.

The participants were then asked which statement was more indicative of their personal understanding using a five-point Likert scale. A list of the survey items for Study 2 is provided in the Appendix. The survey items were initially piloted with both mathematicians and mathematics majors to be sure each group interpreted the strategies in the choices in a way consistent with how the participants implemented these strategies in Study 1.

\section{Results}

A summary of the results is presented in Table 2.

Table 2 provides support for the claim that mathematicians desire that students use all of the strategies with the exception of Strategy 1b. For each of these strategies, the large majority of mathematicians chose Statement A, which stated that they preferred their students use these strategies, and few mathematicians chose statement B, which indicated they preferred their students not use these strategies. Perhaps surprisingly, $23 \%$ of the mathematicians exhibited a preference for Statement B for Strategy 3, which stated

Table 2 Mathematicians' responses to the survey items

\begin{tabular}{llrr}
\hline Strategy & Prefer & Neutral & \multicolumn{2}{c}{$\begin{array}{l}\text { Prefer } \\
\text { B }\end{array}$} \\
\hline 1a. Rephrase theorem in own words & $67.4 \%{ }^{* *}$ & $18.1 \%$ & $14.5 \%$ \\
1b. Write theorem in first-order logic & $19.3 \%$ & $14.5 \%$ & $66.3 \%{ }^{* *}$ \\
2. Attempt to prove theorem before reading its proof & $88.0 \%{ }^{* *}$ & $8.4 \%$ & $3.6 \%$ \\
3. Consider proof frameworks & $63.9 \%{ }^{* *}$ & $13.2 \%$ & $22.9 \%$ \\
4. Break proof into parts & $88.0 \%{ }^{* *}$ & $7.2 \%$ & $4.8 \%$ \\
5. Illustrate assertion with example & $71.1 \%{ }^{* *}$ & $16.9 \%$ & $12.5 \%$ \\
6. Compare proof method with one's own approach & $86.7 \%{ }^{* *}$ & $10.8 \%$ & $2.4 \%$ \\
\hline
\end{tabular}

** - A Wilcoxon one-sample signed-rank test revealed that participants' responses deviated significantly from "neutral" with an alpha-level of 0.05 
they would prefer their students first see how new assertions could be derived from previous statements, rather than Statement A, which stated they would prefer if their students first consider the assumptions and conclusions of the proof before reading it at a line-by-line level. Table 2 offers less support for the claim that mathematicians valued Strategy 1b, where the majority of mathematicians (66\%) preferred their students not use these strategies. It appears that, from the mathematicians' perspective at least, this would not be a productive or appropriate strategy to improve proof comprehension. Strategy $1 \mathrm{~b}$ was only used by the participants in Study 1 when studying Theorem 4 and Proof 4 . It is possible that this strategy may have been beneficial for Theorem 4 and Proof 4, but not for many other theorems and proofs. In a separate study, my colleague and I found that the large majority of mathematics majors claimed to use strategy 1a (Weber \& Mejia-Ramos 2013), so along with Strategy 1b, I also did not include Strategy 1a as a "potentially effective" strategy as the majority of mathematics majors already claimed to use it.

\section{Discussion}

\section{Summary of Results}

In this paper, I have identified five potentially effective strategies that mathematics majors can use to improve their proof comprehension. These strategies include trying to prove a theorem before reading its proof; attending to the assumptions, conclusions, and proof technique in the proof being studied; breaking a long proof into parts; using an example to check confusing assertions within a proof; and comparing the technique used in the proof to one's approach. In the qualitative study, the potential effectiveness of each strategy was illustrated with instances of a pair of students applying this strategy to gain some type of insight. In the survey study, the large majority of mathematicians desired that mathematics majors use these strategies when reading proofs.

\section{Caveats}

The qualitative study that was used to generate the proof reading strategies had a relatively small number of participants (two pairs of students) reading a relatively small number of proofs (six) from two domains (calculus and number theory). It is likely that if other students were interviewed or other proofs were used, different effective proof reading strategies may have emerged. Consequently there is no claim that this paper offers a comprehensive taxonomy of every proving reading strategy. It is probable that there are other strategies, such as drawing a diagram or identifying where each hypothesis in a theorem statement was used in a proof, that would also be useful for developing proof comprehension. What this study does offer is a beginning list of proof reading strategies that may be useful for proof comprehension.

\section{The Relationship between Social Interactions and Proof Reading Strategies}

In the first study, I used a cognitive lens to extrapolate proving strategies from pairs of students' proof-reading activity. Although the role of social interactions was not an explicit topic of investigation, it is interesting to note that working in pairs may have 
facilitated the use of these strategies. For strategy 3, considering the proof framework used in the proof, Kevin's naming the propositions in the theorem statement $\mathrm{P}$ and $\mathrm{Q}$ appeared to be done to facilitate his interaction with Tim by clearly establishing symbols to designate the two assertions in the theorem statement. These symbols were useful in clarifying what the assumptions and conclusions were in each of the subproofs. For strategy 6, Derek and Caleb were jointly marveling at the approach used in Proof 1, which may have facilitated the method itself as becoming an object of discussion. One way that students may come to adopt the strategies in this paper is by internalizing the dialog from social interactions.

\section{Directions for Future Research}

A contribution of this paper is the suggestion that students' understanding of the proofs that they read can be improved if they can be taught to apply the strategies used in this paper. However, the link between identifying these strategies and teaching them to students is not straightforward. There is an interesting analogy between the strategies identified in this paper and the problem-solving heuristics described by Pólya (1957). In the 1970 s and 1980s, mathematics educators tried to improve students' problemsolving performance by explicitly teaching them Polya's heuristics, usually by modeling these heuristics and giving students the opportunities to practice their application. However, such efforts were generally unsuccessful (Begle 1979; Schoenfeld 1985). Designing effective instruction to teach these heuristics required substantial effort, including addressing other areas of students' problem-solving performance such as content knowledge and beliefs as well as having a more prescriptive understanding of how these strategies could be implemented (Schoenfeld 1985). Hence, identifying problem-solving heuristics was an important first step to improving students' problem-solving performance, but more research was needed before they could be incorporated effectively into instruction.

The proof reading strategies identified in this paper suggest a direction for improving mathematics instruction; if students could be led to apply these strategies effectively, the comprehension of the proofs that they read might be improved. However, more research is needed into the fine-grained details as to how these strategies are implemented as well as instructional environments that support students' adaptation of these strategies. My colleagues and I are currently addressing these issues using design research-based teaching experiments (e.g., Cobb et al. 2003), where the proof comprehension strategies and the methods used to teach them are both being evaluated and refined (Samkoff \& Weber 2015). If such instructional efforts are successful, they would form a useful contribution to undergraduate mathematics education research. Students would not only be better able to comprehend the proofs that are presented to them in class, but they may have a deeper appreciation about the nature of proof as well.

Acknowledgments This material is based upon the work supported by the National Science Foundation under grants DUE-1245626 and DRL-0643734. Any opinions, findings, and conclusions or recommendations expressed in this material are those of the authors and do not necessarily reflect the views of the National Science Foundation. I would like to thank Aron Samkoff for assisting with the coding of the data from Study 1 in this paper. 


\section{Appendixes}

\section{Appendix 1. Theorems and Proofs Used in the Qualitative Study}

Theorem 1 Claim: $4 x^{3}-x^{4}+2 \sin x=30$ has no solutions.

Proof 1 Consider the functions $f(x)=4 x^{3}-x^{4}$ and $g(x)=2 \sin x$.

Since $f(x)$ is a polynomial of degree 4 whose leading coefficient is negative, $f(x) \rightarrow$ $-\infty$ as $x \rightarrow \infty$ and $x \rightarrow-\infty$. Hence, $f(x)$ will have an absolute maximum. Taking the first derivative of $f(x)$ yields $f^{\prime}(x)=12 x^{2}-4 x^{3}$. Setting $f^{\prime}(x)$ equal to zero and solving for $x$ yields $x=0$ and $x=3$. These are the critical points of $f(x)$. The absolute maximum must occur at a critical point. $f(0)=0 . f(3)=27$. Hence, $f(x) \leq 27$ for all $x$.

The range of $\sin x$ is $[-1,1]$. Hence the range of $2 \sin x$ is $[-2,2]$. Therefore $g(x)=2$ $\sin x \leq 2$ for all $x .4 x^{3}-x^{4}+2 \sin x=f(x)+g(x) \leq \max (f(x))+\max (g(x))=27+2=29<30$ Therefore, $4 x^{3}-x^{4}+2 \sin x=30$ has no solutions.

Theorem 2 Let $a, b$, and $c$ be natural numbers. $(a, b, c)$ is a Primitive Pythagorean triple if two conditions hold:

(1) $a^{2}+b^{2}=c^{2}$

(2) $a, b$, and c are co-prime (i.e., there is not a common factor of $a, b$, and $c$ other than 1)

For example, $(3,4,5)$ and $(5,12,13)$ are Pythagorean triples. $(9,12,15)$ is not a Pythagorean triple because 3 is a factor of 9,12 , and $15 .(4,5,7)$ is not a Pythagorean triple because $4^{2}+5^{2}=41 \neq 49=7^{2}$.

Theorem: If $(a, b, c)$ is a Primitive Pythagorean triple, then $c$ is odd.

Proof 2

Lemma 1: $a^{2} \equiv 1(\bmod 4)$ if $a$ is odd and $a^{2} \equiv 0(\bmod 4)$ if $a$ is even.

Proof: Suppose $a$ is odd. Then $a=2 k+1$ for some integer k. $a^{2}=(2 k+1)^{2}=4 k^{2}+4$ $k+1=4\left(k^{2}+k\right)+1$. So $a^{2} \equiv 1(\bmod 4)$. Suppose $a$ is even. Then $a=2 k$ for some integer $k$. $a^{2}=4 k^{2}$ so $a$ is divisible by 4 and hence $a^{2} \equiv 0(\bmod 4)$.

Lemma 2: If $a^{2}$ is odd, then $a$ is odd.

Proof by contraposition. It suffices to show that if $a$ is not odd, then $a^{2}$ is not odd. This is equivalent to showing if $a$ is even, then $a^{2}$ is even. Suppose a is even. Then $a^{2}$ is even since the product of two even numbers is even. This proves the claim.

Proof of theorem (by cases).

There are three cases. Either $\mathrm{a}$ and $\mathrm{b}$ are both even, $\mathrm{a}$ and $\mathrm{b}$ are both odd, or $\mathrm{a}$ is even and $b$ is odd (or vice versa). 
Case 1 ( $a$ and $b$ are both even). Since $a$ and $b$ are even, $a^{2}$ and $b^{2}$ are even. Hence $a^{2}+b^{2}$ is even. If $(a, b, c)$ is a Pythagorean triple, $a^{2}+b^{2}=c^{2}$ so $c^{2}$ is even. By lemma 2 , since $c^{2}$ is even, c must be even. Hence, $a, b$, and $c$ are all even and hence all have a factor of 2 . Thus $(a, b, c)$ is not a primitive Pythagorean triple. There are no primitive Pythagorean triples if $a$ and $b$ are both even.

Case 2 ( $a$ and $b$ are both odd). By lemma 1, since $a$ and $b$ are odd, $a^{2} \equiv 1(\bmod$ $4)$ and $b^{2} \equiv 1(\bmod 4)$. Thus, $a^{2}+b^{2} \equiv 2(\bmod 4)$. If $(a, b, c)$ is a Pythagorean triple, $a^{2}+b^{2}=c^{2} \equiv 1(\bmod 4)$. By Lemma 1 , we know that $c^{2}$ cannot be congruent to $2(\bmod 4)$. Thus there are no primitive Pythagorean triples if $a$ and $b$ are both odd.

Case 3. ( $a$ is odd and $b$ is even, or vice versa). Without loss of generality, assume $a$ is odd and $b$ is even. Then $a^{2}$ is odd and $b^{2}$ is even. Hence $a^{2}+b^{2}$ is odd. If $(a, b, c)$ is a Primitive Pythagorean triple, $a^{2}+b^{2}=c^{2}$ is odd. Since $c^{2}$ is odd, by Lemma $2, c$ is odd.

Hence, the only way to a Pythagorean triple can be formed is if $a$ is odd and $b$ is even or $a$ is even and $b$ is odd. In either case, $c$ is necessarily odd.

Theorem 3 We say that $n$ is k-tuply perfect if and only if $\sigma(n)=\sum_{d / n} d=\mathrm{k} n$.

If $\mathrm{n}$ is 3-tuply perfect and 3 does not divide $\mathrm{n}$, then $3 \mathrm{n}$ is 4 -tuply perfect.

\section{Proof 3}

1. Lemma: If $m$ and $n$ are relatively prime, then $\sigma(m n)=\sigma(m) \sigma(n)$.

2. Proof: let the prime factorization of $n$ be $n=p_{1}^{b_{1}} p_{2}^{b_{2}} \cdots p_{k}^{b_{k}}$.

3. Then the divisors of $n$ are all numbers of the form

$$
d=p_{1}^{b_{1}} p_{2}^{b_{2}} \cdots p_{k}^{b_{k}}, \text { where } 0 \leq b_{1} \leq a_{1}
$$

4. But these numbers are precisely the terms in the expansion of the product

$$
\left(1+p_{1}+p_{1}^{2}+\ldots p_{1}^{a_{1}}\right)\left(1+p_{2}+p_{2}^{2}+\ldots p_{2}^{a_{2}}\right) \ldots\left(1+p_{k}+p_{k}^{2}+\ldots p_{k}^{a_{k}}\right)
$$

5. Thus, it must be that

$$
\sigma(n)=\left(1+p_{1}+p_{1}^{2}+\ldots+p_{1}^{a_{1}}\right)\left(1+p_{2}+p_{2}^{2}+\ldots+p_{2}^{a_{2}}\right) \ldots\left(1+p_{k}+p_{k}^{2}+\ldots+p_{k}^{a_{k}}\right)
$$

6. Since each set of parentheses contains a geometric sum, we can rewrite this as

$$
\sigma(n)=\frac{p_{1}^{a_{1}}-1}{p_{1}-1} \frac{p_{1}^{a_{2}}-1}{p_{2}-1} \ldots \frac{p_{k}^{a_{k}}-1}{p_{k}-1} .
$$

7. For $n$ and $m$ relatively prime, we will have

$$
m n=p_{1}^{b_{1}} p_{2}^{b_{2}} \cdots p_{k}^{b_{k}} q_{1}^{b_{1}} q_{2}^{b_{2}} \cdots q_{j}^{b_{j}}, \text { where } n=p_{1}^{b_{1}} p_{2}^{b_{2}} \cdots p_{k}^{b_{k}} \text { and } m=q_{1}^{b_{1}} q_{2}^{b_{2}} \cdots q_{j}^{b_{j}} .
$$


8. Then

$$
\sigma(m n)=\frac{p_{1}^{a_{1}}-1}{p_{1}-1} \frac{p_{2}^{a_{2}}-1}{p_{2}-1} \cdots \frac{p_{k}^{a_{k}}-1}{p_{k}-1} \cdot \frac{q_{1}^{a_{1}}-1}{q_{1}-1} \frac{q_{1}^{a_{2}}-1}{q_{2}-1} \cdots \frac{q_{j}^{a_{j}}-1}{q_{j}-1}=\sigma(m) \sigma(n) .
$$

9. With this lemma in hand, suppose that $\sigma(n)=3 n$ and $n$ is not a multiple of 3 .

10. Then $\sigma(3 n)=\sigma(3) \sigma(n)$.

11. However, $\sigma(3)=1+3=4$, so $\sigma(3 n)=4 \sigma(n)=4(3 n)$, which makes $3 n$ 4-tuply perfect.

Theorem 4 There is a real number whose fourth power is exactly one larger than itself.

\section{Proof 4}

1. Let $f(x)=x^{4}-x-1$.

2. $f(1)=-1$ and $f(2)=13$.

3. By the intermediate value theorem, there must be some number $c$ such that $1<c<2$ and $f(c)=0$.

4. $c$ has the desired property.

Theorem 5 If every even integer greater than 2 can be expressed as the sum of two primes, then every integer greater than 5 can be expressed as the sum of three primes.

\section{Proof 5}

1. Assume every even integer greater than 2 can be expressed as the sum of two primes.

2. Let $n>5$.

3. We proceed by cases.

4. Case 1: $n$ is even.

5. Then $n-2$ is even and greater than 2 .

6. So $n-2=p_{1}+p_{2}$, with $p_{1}$ and $p_{2}$ prime.

7. Then $n=p_{1}+p_{2}+2$, a sum of three primes.

8. Case $2: n$ is odd.

9. Then $n-3$ is even and greater than 2 .

10. So $n-3=p_{1}+p_{2}$, with $p_{1}$ and $p_{2}$ prime.

11. Then $n=p_{1}+p_{2}+3$, a sum of three primes.

Theorem 6 The number of divisors of a positive integer $n$ is odd if and only if $n$ is a perfect square.

\section{Proof 6}

1. Let $d$ be a divisor of $n$.

2. Then $\frac{n}{d}$ is also a divisor of $n$.

3. Suppose $n$ is not a perfect square.

4. Then $\frac{n}{d} \neq d$ for all divisors $d$, so we can pair up all divisors by pairing $d$ with $\frac{n}{d}$.

5. Thus, $n$ has an even number of divisors. 
6. On the other hand, suppose $n$ is a perfect square.

7. Then $\frac{n}{d}=d$ for some divisor $d$.

8. In this case, when we pair divisors by pairing $d$ with $\frac{n}{d}, d$ will be left out, so $n$ has an odd number of divisors.

\section{Appendix 2. Survey Items for Study 2}

Strategy \#1: Understanding the theorem statement before reading its proof

A. Before mathematics majors read a proof of a theorem, I would prefer that they try to describe the theorem in their own words.

B. I would not mind if mathematics majors did not usually describe the theorem in their own words before reading its proof.

A. Before reading a proof of a theorem, I would prefer if mathematics majors expressed the theorem in logical notation to see what needs to be proven.

B. I would not mind if mathematics majors did not usually express theorems in logical notation before reading its proof.

Strategy \#2: Trying to prove a theorem before reading its proof

A. When reading a proof of a theorem, I would prefer if mathematics majors think about how they might prove the theorem themselves BEFORE reading the proof.

B. I would prefer that mathematics majors not try to prove a theorem themselves before reading its proof. A reason for reading the proof is to see why the theorem is true.

Strategy \#3: Considering the proof framework being used in the proof

A. When mathematics majors read a proof, I would prefer if they first consider is what is being assumed, what is being concluded, and what proof technique is being used.

B. When mathematics majors read a proof, I would prefer if they first consider how each new statement can be derived from previous statements.

\section{Strategy \#4: Breaking a long proof into parts or sub-proofs}

A. When mathematics majors read a long proof, I would prefer if they try to break it into parts or sub-proofs.

B. When mathematics majors read a long proof, I would prefer if they do not break it into parts but try to understand how each line follows from previous assertions. 


\section{Strategy \#5: Checking assertions within the proof with examples}

A. When mathematics majors read a new assertion in a proof that they find confusing, I would prefer if they sometimes checked whether that assertion is true with a numerical example.

B. When mathematics majors read a new assertion in a proof, I would prefer if they try to see how that new assertion is a logical consequence of previous statements. They should not check assertions with specific examples since you cannot prove by example.

\section{Strategy \#6: Comparing the method in the proof to one's own methods}

A. When mathematics majors read a proof, I would prefer if they compare how the method used in the proof compares to the method they might use to prove the theorem.

B. When mathematics majors read a proof, I would prefer if they try not to consider how they might approach the proof.

\section{References}

Alcock, L. (2009). e-Proofs: Students experience of online resources to aid understanding of mathematical proofs. In Proceedings of the 12th Conference for Research in Undergraduate Mathematics Education. Retrieved from http://sigmaa.maa.org/rume/crume2009/proceedings.html.

Alcock, L., \& Simpson, A. (2004). Convergence of sequences and series: interactions between visual reasoning and the learner's beliefs about their own role. Educational Studies in Mathematics, 57, 1-32.

Alcock, L., \& Weber, K. (2005). Proof validation in real analysis: inferring and evaluating warrants. Journal of Mathematical Behavior, 24(2), 125-134.

Anderson, J., Boyle, C., \& Yost, G. (1986). The geometry tutor. Journal of Mathematical Behavior, 5, 5-20.

Begle, E. G. (1979). Critical variables in mathematics education: findings from a survey of the empirical literature. Washington, DC: Mathematical Association of America and the National Council for Teachers of Mathematics.

Cobb, P., Confrey, J., diSessa, A., Lehrer, R., \& Schauble, L. (2003). Design experiments in educational research. Educational Researcher, 32(1), 9-13.

Conradie, J., \& Frith, J. (2000). Comprehension tests in mathematics. Educational Studies in Mathematics, $42(3), 225-235$.

Cowen, C. (1991). Teaching and testing mathematics reading. American Mathematical Monthly, 98(1), 50-53.

Davis, P. J., \& Hersh, R. (1981). The mathematical experience. New York: Viking Penguin Inc.

de Villiers, M. (1990). The role and function of proof in mathematics. Pythagoras, 24, 17-24.

Dreyfus, T. (1991). Advanced mathematical thinking processes. In D. Tall (Ed.), Advanced Mathematical Thinking (pp. 25-41). Dordrecht: Kluwer.

Dreyfus, T., Nardi, E., \& Leikin, R. (2012). Forms of proof and proving in the classroom. In G. Hanna \& M. de Villiers (Eds.), Proof and proving in mathematics education: The 19th ICMI study (pp. 191-213). Dordrecht: Springer.

Firestone, W. (1993). Alternative arguments for generalizing from data as applied to qualitative research. Educational Researcher, 22, 16-23.

Fukawa-Connelly, T. (2012). A case study of one instructor's lecture-based teaching of proof in abstract algebra: making sense of her pedagogical moves. Educational Studies in Mathematics, 81, 325-345.

Fuller, E., Weber, K., Mejía-Ramos, J. P., Samkoff, A., \& Rhoads, K. (2014). Comprehending structured proofs. International Journal of Studies in Mathematics Education, 7, 1-32. 
Gosling, S. D., Vazire, S., Srivastava, S., \& John, O. P. (2004). Should we trust web-based studies? A comparative analysis of six studies about internet questionnaires. American Psychologist, 59, 93-104.

Hanna, G. (1990). Some pedagogical aspects of proof. Interchange, 21(1), 6-13.

Hanna, G., \& Barbeau, E. (2010). Proofs as bearers of mathematical knowledge. In G. Hanna, H. N. Jahnke, \& H. Pulte (Eds.), Explanation and proof in mathematics (pp. 85-100). Dordrecht: Springer.

Harel, G., \& Sowder, L. (1998). Students' proof schemes: Results from exploratory studies. In A. Schoenfeld, J. Kaput, \& E. Dubinsky (Eds.), Research in Collegiate Mathematics Education III (pp. 234-283). Providence, RI: American Mathematical Society.

Healy, L., \& Hoyles, C. (2000). A study of proof conceptions in algebra. Journal for Research in Mathematics Education, 31, 396-428.

Hemmi, K. (2008). Students' encounter with proof: the condition of transparency. ZDM- The International Journal on Mathematics Education, 40, 413-426.

Hemmi, K., \& Jaworski, B. (2009). Transparency in a student-tutor interaction concerning the converse of Lagrange's Theorem. In F. L. Lin, F. J. Hsieh, G. Hanna, \& M. de Villiers (Eds.), ICMI Study 19: Proof and proving in mathematics education (Vol. 1, pp. 202-207). Taipei: The Department of Mathematics, National Taiwan Normal University.

Hodds, M., Alcock, L., \& Inglis, M. (2014). Self-explanation training improves proof comprehension. Journal for Research in Mathematics Education, 45, 62-101.

Inglis, M., \& Alcock, L. (2012). Expert and novice approaches to reading mathematical proofs. Journal for Research in Mathematics Education, 43(4), 358-391.

Inglis, M., \& Mejia-Ramos, J. P. (2009). The effect of authority on the persuasiveness of mathematical arguments. Cognition and Instruction, 27, 25-50.

Ko, Y., \& Knuth, E. (2012). Validating proofs and counterexamples across domains: practices of importance for mathematics majors. Journal of Mathematical Behavior, 32, $20-35$.

Kranz, J., \& Dalal, R. (2000). Validity of web-based psychological research. In M. Birnbaum (Ed.), Psychological experiments on the internet (pp. 35-60). San Diego: Academic.

Lai, Y., \& Weber, K. (2014). Factors mathematicians profess to consider when presenting pedagogical proofs. Educational Studies in Mathematics, 85, 93-108.

Lai, Y., Weber, K., \& Mejía-Ramos, J. P. (2012). Mathematicians' perspectives on features of a good pedagogical proof. Cognition and Instruction, 30, 146-169.

Leron, U. (1983). Structuring mathematical proofs. American Mathematical Monthly, 90(3), 174-184.

Leron, U., \& Zaslavsky, O. (2009). Generic proving: Reflections on scope and method. In F. L. Lin, F. J. Hsieh, G. Hanna, \& M. de Villiers (Eds.), ICMI Study 19: Proof and proving in mathematics education (Vol. 2, pp. 53-58). Taipei: The Department of Mathematics, National Taiwan Normal University.

Martin, W. G., \& Harel, G. (1989). Proof frames of pre-service elementary teachers. Journal for Research in Mathematics Education, 20(1), 41-51.

Mejía-Ramos, J. P., Fuller, E., Weber, K., Rhoads, K., \& Samkoff, A. (2012). An assessment model for proof comprehension in undergraduate mathematics. Educational Studies in Mathematics, 79(1), 3-18.

Mejía-Ramos, J. P., and Inglis, M. (2009). Argumentative and proving activities in mathematics education research. In F.-L. Lin, F.-J. Hsieh, G. Hanna, \& M. de Villiers (Eds.), Proceedings of the ICMI Study 19 conference: Proof and Proving in Mathematics Education (Vol. 2, pp. 88-93). Taipei: The Department of Mathematics, National Taiwan Normal University.

Mejía-Ramos, J. P., \& Weber, K. (2014). How and why mathematicians read proofs: further evidence from a survey study. Educational Studies in Mathematics, 85, 161-173.

Mills, M. (2011). Mathematicians' pedagogical thoughts and practices in proof presentation. Presentation at the 14th Conference for Research in Undergraduate Mathematics Education. Abstract retrieved from http://sigmaa.maa.org/rume/crume2011/Preliminary_Reports.html.

Nardi, E. (2008). Amongst mathematicians: Teaching and learning mathematics at university level. New York, NY: Springer.

Nardi, E. (2011). Driving noticing yet 'risking precision': University mathematicians' pedagogical perspectives on verbalisation in mathematics. In M. Pytlak, T. Rowland, \& E. Swoboda (Eds.), Proceedings of the 7th Conference on European Research in Mathematics Education (pp. 2053-2062). Rzeszow: University of Rzeszow.

Pólya, G. (1957). How to solve it: a new aspect of mathematical method. Princeton, NJ: Princeton University Press.

Porteous, K. (1986). Children's appreciation of the significance of proof. In Proceedings of the Tenth International Conference for the Psychology of Mathematics Education (pp. 392-397). London: University of London, Institute of Education.

Rav, Y. (1999). Why do we prove theorems? Philosophia Mathematica, 7(3), 5-41.

Rowland, T. (2001). Generic proofs in number theory. In S. Campbell \& R. Zazkis (Eds.), Learning and teaching number theory: Research in cognition and instruction (pp. 157-184). Westport, CT: Ablex. 
Samkoff, A. \& Weber, K. (2015). Lessons learned from an instructional intervention on proof comprehension. To appear in The Journal of MathematicalBehavior, 39, 28-50.

Schoenfeld, A. (1985). Mathematical Problem Solving. Orlando: Academic.

Segal, J. (2000). Learning about mathematical proof: conviction and validity. Journal of Mathematical Behavior, 18, 191-210.

Selden, A. (2012). Transitions and proof and proving at the tertiary level. In G. Hanna \& M. de Villiers (Eds.), Proof and proving in mathematics education: The 19th ICMI study (pp. 391-420). Dordrecht: Springer.

Selden, A., \& Selden, J. (2003). Validations of proofs considered as texts: can undergraduates tell whether an argument proves a theorem? Journal for Research in Mathematics Education, 34(1), 4-36.

Selden, J., \& Selden, A. (1995). Unpacking the logic of mathematical statements. Educational Studies in Mathematics, 29(2), 123-151.

Strauss, A., \& Corbin, J. (1990). Basics of qualitative research: grounded theory procedures and techniques. London: SAGE.

Weber, K. (2004). Traditional instruction in advanced mathematics: a case study of one professor's lectures and proofs in an introductory real analysis course. Journal of Mathematical Behavior, 23, 115-133.

Weber, K. (2008). How mathematicians determine if an argument is a valid proof. Journal for Research in Mathematics Education, 39, 431-459.

Weber, K. (2010). Mathematics majors perceptions of conviction, validity, and proof. Mathematical Thinking and Learning, 12, 303-336.

Weber, K. (2012). Mathematicians' perspectives on their pedagogical practice with respect to proof. International Journal of Mathematics Education in Science and Technology, 43(4), 463-482.

Weber, K., \& Alcock, L. (2005). Using warranted implications to understand and validate proofs. For the Learning of Mathematics, 25(1), 34-38.

Weber, K., Inglis, I., \& Mejía-Ramos, J. P. (2014). How mathematicians obtain conviction: implications for mathematics instruction and epistemic cognition. Educational Psychologist, 49, 36-58.

Weber, K., \& Mejía-Ramos, J. P. (2011). Why and how mathematicians read proofs: an exploratory study. Educational Studies in Mathematics, 76(3), 329-344.

Weber, K., \& Mejía-Ramos, J. P. (2013). Effective but underused strategies for proof comprehension. In M. Martinez \& A. Castro Superfine (Eds.), Proceedings of the 35th Annual Meeting for the North American Chapter of the Psychology of Mathematics Education (pp. 260-267). Chicago, IL: University of Illinois at Chicago.

Weber, K., \& Mejía-Ramos, J. P. (2014). Mathematics majors' beliefs about proof reading. International Journal of Mathematics Education in Science and Technology, 45, 89-103.

Weber, K. \& Mejía-Ramos, J.P. (2015). The contextual nature of conviction in mathematics. To appear in For the Learning of Mathematics, 35(2).

Yopp, D. (2011). How some research mathematicians and statisticians use proof in undergraduate mathematics. Journal of Mathematical Behavior, 30, 115-130. 